\title{
Percepción sobre políticas de espacios libres de humo de tabaco en bares y restaurantes del centro de México
}

\author{
Tonatiuh Barrientos-Gutiérrez, PhD, (1) David Gimeno, PhD, (2,3) James F Thrasher, PhD, (1,4) \\ Luz Myriam Reynales-Shigematsu, ScD, (1) Benjamin C Amick III, PhD, (3,5) Eduardo Lazcano-Ponce, ScD, (1) \\ Mauricio Hernández-Ávila, PhD.(6)
}

\begin{abstract}
Barrientos-Gutiérrez T, Gimeno D, Trasher JF, Reynales-Shigematsu LM, Amick III BC, Lazcano-Ponce E, Hernández-Ávila M. Percepción sobre políticas de espacios libres de humo de tabaco en bares y restaurantes del centro de México. Salud Publica Mex 2010;52 supl 2:SI49-SI56.
\end{abstract}

\section{Resumen}

Objetivo. Analizar las percepciones y apreciaciones sobre espacios libres de humo de tabaco de los responsables de bares y restaurantes en cuatro ciudades del centro de México. Material y métodos. Se encuestó a los administradores de 219 bares y restaurantes de la Ciudad de México, Colima, Cuernavaca y Toluca, acerca de opiniones e implementación de espacios libres de humo. Simultáneamente, se monitorearon concentraciones de nicotina ambiental. Resultados. La mayoría de los encuestados consideró que los espacios públicos deben ser libres de humo, más de la mitad refirió preocupación por pérdidas económicas. La implementación de espacios libres de humo fue más frecuente en la Ciudad de México ( $85.4 \%$ ) que en las demás ciudades (I5.3\% global), lo que se refleja en menores concentraciones de nicotina ambiental. Conclusión. Los administradores reconocen la necesidad de crear espacios libres de humo. La preocupación sobre posibles efectos económicos negativos de la prohibición podría explicar, al menos parcialmente, el rechazo a la implementación de este tipo de políticas.

Palabras clave: humo de tabaco ambiental; bares y restaurantes; legislación; cumplimiento; México

\author{
Barrientos-Gutiérrez T, Gimeno D, Trasher JF, \\ Reynales-Shigematsu LM, Amick III BC, \\ Lazcano-Ponce E, Hernández-Ávila M. \\ Perception over smoke-free policies amongst bar \\ and restaurant representatives in central Mexico. \\ Salud Publica Mex 2010;52 suppl 2:SI49-SI56.
}

\begin{abstract}
Objective. To analyze the perceptions and appreciations over smoke-free environments of restaurant and bar managers from four cities in central Mexico. Material and Methods. Managers from 219 restaurants and bars from Mexico City, Colima, Cuernavaca and Toluca were surveyed about smoke-free environments opinions and implementation. Simultaneously, environmental nicotine was monitored. Results. The majority of surveyed managers considered public places should be smoke-free, although more than half were concerned with potential economic loses. Implementation of smoke-free environments was more frequent in Mexico City $(85.4 \%)$ than in the other cities ( $15.3 \%$ overall), with consequently lower environmental nicotine concentrations. Conclusion. Managers acknowledge the need to create smoke-free environments. Concerns over economic negative effects derived from the prohibition could explain, at least partially, the rejection of this sector towards the implementation of this type of policy.
\end{abstract}

Keywords: environmental tobacco smoke; restaurants and bars; policy; compliance; Mexico

(I) Centro de Investigación en Salud Poblacional. Instituto Nacional de Salud Pública. Cuernavaca, Morelos, México.

(2) The University of Texas Health Science Center at Houston, School of Public Health, San Antonio Campus. Texas, USA.

(3) Institute for Work and Health. Toronto, Canada.

(4) Department of Health Promotion, Education and Behavior. University of South Carolina. South Carolina, USA.

(5) The University of Texas Health Science Center at Houston, School of Public Health. Texas, USA.

(6) Secretaría de Salud. México.

Fecha de recibido: 26 de marzo de $2010 \cdot$ Fecha de aceptado: 12 de julio de 2010

Solicitud de sobretiros: Dr. Tonatiuh Barrientos Gutiérrez. Departamento de Investigación sobre Tabaco. Centro de Investigación en Salud Poblacional. Instituto Nacional de Salud Pública. 7a cerrada de Fray Pedro de Gante 50. Col. Sección XVI, I4000 Delegación Tlalpan, México, DF.

Correo electrónico: tbarrientos@correo.insp.mx 
T a eliminación de la exposición al humo de tabaco ـambiental (HTA) en los lugares públicos es un objetivo prioritario de salud pública, respaldado internacionalmente por más de 160 países firmantes del Convenio Marco para el Control del Tabaco de la Organización Mundial de la Salud. ${ }^{1}$ La eliminación de los daños a la salud derivados de la exposición al HTA requiere del desarrollo de espacios $100 \%$ libres de humo, objetivo que sólo puede lograrse a través de la prohibición completa del consumo de tabaco en áreas interiores. ${ }^{2}$ En la última década diversos países han implementado políticas que prohíben el consumo en áreas interiores de lugares públicos, reduciendo exitosamente la exposición..$^{3-7}$

Los bares y los restaurantes representan espacios críticos para el control del HTA, ya que son establecimientos de alta afluencia y han reportado concentraciones muy elevadas de compuestos derivados del tabaco en el ambiente, ${ }^{8,9}$ absorbidos con facilidad por clientes y trabajadores. ${ }^{10,11}$ Dado que el HTA es causa de enfermedad cardiovascular y cáncer de pulmón, se espera un incremento de 20 a 30\% en el riesgo de estas enfermedades entre los trabajadores y clientes expuestos en bares y restaurantes. ${ }^{12}$ Sin embargo, los bares y restaurantes, manipulados por las compañías tabacaleras, se han opuesto frecuentemente a la implementación de espacios $100 \%$ libres de humo de tabaco, bajo el argumento de supuestos efectos económicos adversos. ${ }^{13}$

El 3 de abril de 2008 entró en vigor la Ley de Protección a la Salud de los No Fumadores del Distrito Federal (LPSNF-DF), estableciendo la prohibición completa al consumo de tabaco en áreas interiores de todos los espacios públicos. ${ }^{14}$ La prohibición al consumo de tabaco en bares y restaurantes mantuvo un alto nivel de aprobación entre la población general. ${ }^{15} \mathrm{~A}$ pesar de que la experiencia en otros países mostraba que la implementación de prohibiciones al consumo de tabaco no tenía efectos sobre el sector hostelero, ${ }^{16}$ la Cámara Nacional de la Industria de Restaurantes y Alimentos Condimentados (CANIRAC) reportaba una posible caída de entre 10 y 30\% en el ingreso del sector. ${ }^{17}$ Bajo esta expectativa, evidencia anecdótica mostraba una alta impopularidad de la LPSNF-DF entre los dueños de bares y restaurantes.

La LPSNF-DF establece que los representantes legales de los establecimientos son corresponsables en el cumplimiento de la Ley, debiendo exhortar a los clientes a no fumar en áreas interiores y solicitando el apoyo de la autoridad competente en caso de negativa reiterada. Por esta razón, el apoyo y la participación activa de los dueños y administradores de bares y restaurantes son fundamentales para la implementación efectiva de la LPSNF-DF. Sin embargo, hasta el momento ningún estudio ha evaluado de manera sistemática la opinión y apreciación de los responsables de bares y restaurantes hacia el desarrollo de espacios 100\% libres de humo de tabaco en México.

El presente artículo describe los resultados de una encuesta aplicada a dueños y administradores de bares y restaurantes de las ciudades de México, Colima, Cuernavaca y Toluca, cuatro meses después de la implementación de la LPSNF-DF. Se analiza la opinión de dueños y administradores sobre la exposición al HTA y los posibles efectos de la prohibición sobre sus establecimientos, comparando los hallazgos de la Ciudad de México con las ciudades sin prohibición. Adicionalmente, se correlaciona el reporte de implementación de políticas para el consumo de tabaco para cada ciudad con las concentraciones de nicotina ambiental medidas en los establecimientos.

\section{Material y métodos}

\section{Selección de establecimientos}

Cuatro ciudades, representando diferentes niveles de prevalencia de tabaquismo, fueron seleccionadas a conveniencia: Colima (11.5\%), Cuernavaca $(21.7 \%)$, Toluca $(27.5 \%)$ y la Ciudad de México $(27.8 \%) .{ }^{18} \mathrm{Al}$ momento del estudio la Ciudad de México era la única con una prohibición completa al consumo de tabaco en bares y restaurantes. En cada ciudad se obtuvo el censo oficial de bares y restaurantes proporcionado por las autoridades municipales. El censo incluía todos los establecimientos registrados en la ciudad, excepto en la Ciudad de México, donde se utilizó el censo del centro histórico. En cada ciudad los establecimientos se estratificaron por tipo de negocio (bar o restaurante) y tamaño (mayores o menores a $100 \mathrm{~m}^{2}$ ), seleccionando 33 establecimientos al azar de cada estrato con el objetivo de reclutar 13 para el estudio (52 establecimientos por ciudad). Se invitó a un total de 371 establecimientos entre julio y octubre de 2008, de los cuales 219 (60\%) aceptaron participar. Se excluyeron tres establecimientos por falta de datos. Así, la muestra final por ciudad fue de: 66 establecimientos en Colima, 51 en Cuernavaca, 48 en la Ciudad de México y 51 en Toluca. Todos los establecimientos participantes proporcionaron su aprobación escrita para llevar a cabo los procedimientos del estudio. Los procedimientos del estudio fueron aprobados por el Comité para la Protección de Sujetos Humanos de la Universidad de Texas (HSCSPH-07-0384) y por los Comités de Ética, Investigación y Bioseguridad del Instituto Nacional de Salud Pública de México (1456-6307-0). 


\section{Encuesta de opinión}

Los dueños o administradores de los establecimientos participantes contestaron una encuesta autoaplicada que contenía información sobre características del establecimiento, opiniones y apreciaciones sobre los espacios libres de humo y políticas sobre el consumo de tabaco.

El nivel económico del vecindario fue evaluado con la pregunta: "Siendo 10 el más rico y 1 el más pobre, ¿cómo calificaría el nivel económico del vecindario donde está su compañía?" Para el análisis se mantuvo la variable de forma continua. La ocupación máxima del local fue definida como el número máximo de personas que pueden permanecer en el local de acuerdo con la licencia de funcionamiento del establecimiento. Los dueños o administradores proporcionaron información sobre su clientela, que incluyó edad promedio en años, escolaridad, composición por género y proporción que fumaba dentro del establecimiento. El nivel de HTA percibido por el dueño o administrador fue establecido con la pregunta "En promedio, ¿qué tan alto es el nivel de humo de tabaco ambiental en su local?", con posibilidades de respuesta: "muy alto", "alto", "medio", "bajo", "muy bajo", "nulo". Para el análisis se crearon tres categorías: "nulo", "bajo" (colapsa "bajo" y "muy bajo") y alto (colapsa "medio", "alto" y "muy alto"). El tipo de establecimiento fue definido con la pregunta "¿Es este establecimiento un restaurante o un bar?" con posibilidades de respuesta "bar", "restaurante" y "restaurante-bar". En el caso de los restaurantes-bar se pidió a los dueños o administradores que lo clasificaran como "bar" o "restaurante" en función de la actividad principal.

Las opiniones y apreciaciones de los dueños y administradores fueron recuperadas a partir de dos grupos de preguntas. El primer grupo pedía que expresaran su opinión a las preguntas: “iLos lugares públicos deberían ser considerados espacios libres de humo de tabaco?", "¿Los bares y restaurantes deberían ser considerados espacios libres de humo de tabaco?" y "¿El humo de tabaco ambiental es nocivo para la salud de los no fumadores?", con posibles respuestas "Sí", "No" y "No sé". El segundo grupo estaba constituido por las aseveraciones "Si su establecimiento fuera libre de humo de tabaco el número de clientes..." y "Si su establecimiento fuera libre de humo de tabaco el ingreso económico...", con posibles respuestas "aumentaría", "disminuiría" y "no sería afectado", además de las aseveraciones "Si su establecimiento fuera libre de humo la salud de mis clientes..." y "Si su establecimiento fuera libre de humo la salud de sus trabajadores..." con posibles respuestas "mejoraría", "empeoraría" y "no sería afectada". La aseveración "Si su establecimiento fuera libre de humo de tabaco el ingreso económico..." estuvo altamente correlacionada con la aseveración sobre el número de clientes $(r>0.90)$, por lo que se decidió utilizar solamente ésta última.

La política hacia el consumo de tabaco en áreas interiores implementada en el establecimiento fue examinada con la pregunta "¿Cuál es la política de su compañía hacia el consumo de tabaco de sus clientes?" con posibles respuestas: "No pueden fumar en ningún lugar de este local", "Pueden fumar en áreas designadas" y "Pueden fumar en cualquier área que deseen".

\section{Monitoreo de nicotina ambiental}

La medición de nicotina ambiental se llevó a cabo durante el turno de máxima ocupación de clientes, utilizando monitores pasivos de nicotina. Los monitores fueron colocados en el centro de las áreas de fumar y no fumar, en caso de estar ambas disponibles. Para efectos de control de calidad se colocaron $10 \%$ de monitores blancos y 10\% de duplicados. Las concentraciones de nicotina fueron obtenidas por cromatografía de gases con detección selectiva de nitrógeno en las instalaciones del Laboratorio Analítico de Compuestos del Tabaco del Instituto Nacional de Salud Pública de México (concentración mínima detectable $0.03 \mu \mathrm{g} / \mathrm{m}^{3}$ ).

\section{Análisis estadístico}

Se llevó a cabo el análisis estadístico de los datos considerando la población total de establecimientos y estratificando por ciudad. La descripción de variables continuas se realizó calculando la mediana y los percentiles 25 y 75, dada la no normalidad de los datos. Para variables categóricas se calcularon frecuencias y porcentajes. Para evaluar posibles diferencias entre ciudades se utilizó ji cuadrada de Pearson o prueba exacta de Fisher. Las concentraciones de nicotina fueron estratificadas por ciudad evaluando diferencias entre establecimientos en función de las políticas de consumo de tabaco implementadas utilizando la prueba de medianas. Todos los análisis fueron llevados a cabo en Stata 10.1 (College Station, TX).

\section{Resultados}

El cuadro I presenta las características principales de los establecimientos. Los establecimientos reportaron en promedio una capacidad máxima de 80 personas, con edad de 35 años y mayoritariamente hombres $(60 \%)$. El $30 \%$ de los clientes fumaba dentro del establecimiento, salvo en la Ciudad de México, donde la mediana fue 
de $0 \%$. La escolaridad de los clientes reportada más frecuentemente fue "profesionista" (49\%), seguido de "preparatoria" $(24.1 \%)$. La escolaridad promedio fue menor en los establecimientos de la Ciudad de México. Una cuarta parte de los establecimientos consideró que el nivel de HTA en su local era nulo, con $46.8 \%$ en nivel "bajo" y $29 \%$ en "alto". Se observó el mismo patrón en todas las ciudades, excepto en la Ciudad de México, donde dos terceras partes reportaron nivel "nulo".

Las opiniones de los dueños y administradores acerca del HTA y la implementación de espacios 100\% libres de humo se presentan en el cuadro II. La mayoría de los establecimientos $(69.4 \%)$ consideró que los espacios públicos deben ser libres de humo de tabaco, siendo la Ciudad de México la que menor apoyo mostró hacia los espacios públicos libres de humo (54.2\%, $p<0.05)$. El apoyo hacia bares y restaurantes libres de humo de tabaco contó con la aprobación de $47.2 \%$ de los establecimientos, siendo ligeramente mayor en Colima $(60.6 \%)$. Casi todos (94\%) los dueños y administradores consideraron que el HTA es nocivo para la salud. Más de la mitad (57.4\%) de ellos consideraron que la implementación de espacios libres de humo disminuiría el número de clientes de su establecimiento. La mayoría de los establecimientos consideró que la implementación de espacios libres de humo mejoraría la salud de sus clientes $(61.1 \%)$ y trabajadores (65.7\%).

Las políticas implementadas en los establecimientos variaron significativamente por ciudad. Mientras que en Colima, Cuernavaca y Toluca en promedio $15.3 \%$ de los establecimientos prohibía el consumo de tabaco en áreas interiores, en la Ciudad de México el porcentaje fue de $85.4 \%$. En Colima, Cuernavaca y Toluca se detectaron diferencias estadísticamente significativas $(p<0.05)$ en las concentraciones de nicotina entre establecimientos donde se podía fumar libremente, de manera restringida o donde no se podía fumar. En todos los casos las concentraciones fueron menores en los establecimientos donde no se permitía el consumo de tabaco (ver figura 1).

\section{Discusión}

El objetivo de este artículo fue examinar las opiniones y apreciaciones de los dueños de bares y restaurantes acerca del HTA y el desarrollo de espacios 100\% libres de humo de tabaco. La mayoría de los encuestados consideró que el HTA es dañino y que los espacios públicos deben ser libres de humo de tabaco. Sin embargo, sólo la

\section{Cuadro I}

Características de bares y restaurantes participantes. México, 2008

\begin{tabular}{|c|c|c|c|c|c|c|}
\hline Característica & Total $(n=2 / 6)$ & $\begin{array}{l}\text { Colima } \\
(n=66)\end{array}$ & $\begin{array}{c}\text { Cuernavaca } \\
(n=5 I)\end{array}$ & $\begin{array}{l}\text { Ciudad } \\
\text { de México } \\
\text { (n=48) }\end{array}$ & $\begin{array}{l}\text { Toluca } \\
(n=5 I)\end{array}$ & Valor $p$ \\
\hline Nivel económico del vecindario (med., 25-75) & $7(6-8)$ & $7(6-8)$ & $7(6-8)$ & $6(5-8)$ & $7(7-8)$ & 0.029 \\
\hline Ocupación máxima del local (med., 25-75) & $80(45.3-140)$ & $90(49-200)$ & $80(40-140)$ & $80(50-127.5)$ & $70(40-100)$ & 0.054 \\
\hline Edad promedio de los clientes (med., 25-75) & $35(30-40)$ & $35(29.5-40)$ & $35(30-40)$ & $35(35-40)$ & $33(25-40)$ & 0.009 \\
\hline Porcentaje de clientes hombres (med., 25-75) & $60(50-70)$ & $60(50-70)$ & $60(50-70)$ & $60(60-78.8)$ & $60(50-70)$ & 0.159 \\
\hline Porcentaje de clientes que fuma en el local (med., 25-75) & $30(0-70)$ & $45(18.8-75)$ & $40(10-70)$ & $0(0-6.2)$ & $50(0-80)$ & $<0.001$ \\
\hline \multicolumn{7}{|l|}{ Escolaridad de clientes $(\mathrm{n}, \%)$} \\
\hline Primaria/secundaria & $21(9.8)$ & II (16.7) & I $(2.0)$ & $8(16.7)$ & I (9.7) & $<0.001$ \\
\hline Preparatoria & $52(24.1)$ & $16(24.2)$ & $13(25.5)$ & II (22.9) & $12(23.5)$ & \\
\hline Técnico & $37(17.1)$ & $12(18.1)$ & $8(15.7)$ & $10(20.8)$ & $7(13.7)$ & \\
\hline Profesional & $106(49.0)$ & $27(40.9)$ & $29(56.9)$ & $19(39.6)$ & $31(60.8)$ & \\
\hline \multicolumn{7}{|l|}{ Nivel de humo de tabaco en el local $(n, \%)$} \\
\hline Nulo & $52(24.0)$ & $8(12.1)$ & $8(15.7)$ & $32(66.7)$ & $4(7.8)$ & $<0.001$ \\
\hline Bajo & I0I (46.8) & $36(54.6)$ & $29(58.9)$ & $13(27.0)$ & $23(45.1)$ & \\
\hline Alto & $63(29.2)$ & $22(22.3)$ & $14(27.4)$ & $3(6.3)$ & $24(47.1)$ & \\
\hline \multicolumn{7}{|l|}{ Tipo de establecimiento $(n, \%)$} \\
\hline Restaurante & $123(56.9)$ & $30(45.5)$ & $33(64.7)$ & $32(66.7)$ & $28(54.9)$ & 0.085 \\
\hline Bar & $93(43.1)$ & $36(54.5)$ & $18(35.3)$ & $16(33.3)$ & $23(45 . I)$ & \\
\hline
\end{tabular}

(med., 25-75): Mediana, percentil 25-75.

(n, \%): Frecuencia, porcentaje 


\section{CuAdro II}

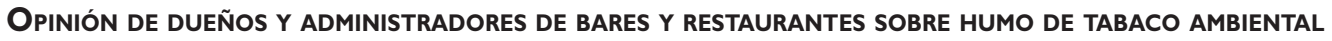
Y DeSARRollo de espacios I00\% libres de humo de tabaco. MéXICO, 2008

\begin{tabular}{|c|c|c|c|c|c|c|}
\hline Opiniones y apreciaciones & $\begin{array}{l}\text { Total* } \\
(n=2 / 6)\end{array}$ & $\begin{array}{l}\text { Colima } \\
(n=66)\end{array}$ & $\begin{array}{c}\text { Cuernavaca } \\
(n=5 I)\end{array}$ & $\begin{array}{c}\text { Ciudad } \\
\text { de México } \\
(n=48)\end{array}$ & $\begin{array}{l}\text { Toluca } \\
(\mathrm{n}=5 I)\end{array}$ & Valor $p$ \\
\hline
\end{tabular}

¿Los lugares públicos deberían ser considerados espacios libres de humo de tabaco?

\begin{tabular}{rllllll} 
Sí & $150(69.4)$ & $53(80.3)$ & $36(70.6)$ & $26(54.2)$ & $35(68.6)$ & 0.030 \\
No / no sé & $66(30.6)$ & $13(19.7)$ & $15(29.4)$ & $22(45.8)$ & $16(31.4)$ & \\
\hline
\end{tabular}

\begin{tabular}{|c|c|c|c|c|c|c|}
\hline \multicolumn{7}{|l|}{$\begin{array}{l}\text { ¿Bares y restaurantes deberían ser considerados espacios } \\
\text { libres de humo de tabaco? }\end{array}$} \\
\hline Si & $102(47.2)$ & $40(60.6)$ & $20(39.2)$ & $19(39.6)$ & $23(45.1)$ & 0.065 \\
\hline No / no sé & II 4 (52.8) & $26(39.3)$ & $31(60.8)$ & $29(60.4)$ & $28(54.9)$ & \\
\hline \multicolumn{7}{|l|}{$\begin{array}{l}\text { ¿El humo de tabaco ambiental es nocivo para la salud de } \\
\text { los no fumadores? }\end{array}$} \\
\hline Si & $203(94.0)$ & $62(94.0)$ & $47(92.2)$ & $46(95.8)$ & $48(94.1)$ & 0.961 \\
\hline No / no sé & $13(6.0)$ & $4(6.0)$ & $4(7.8)$ & $2(4.2)$ & $3(5.9)$ & \\
\hline \multicolumn{7}{|l|}{$\begin{array}{l}\text { Si su establecimiento fuera libre de humo de tabaco el } \\
\text { número de clientes... }\end{array}$} \\
\hline Aumentaría / no afectaría & $92(42.6)$ & $27(41.0)$ & $24(47.1)$ & $25(52.1)$ & $16(31.4)$ & 0.181 \\
\hline Disminuiría & I24 (57.4) & $39(59.0)$ & $27(52.9)$ & $23(47.9)$ & $35(68.6)$ & \\
\hline \multicolumn{7}{|l|}{$\begin{array}{l}\text { Si su establecimiento fuera libre de humo de tabaco la } \\
\text { salud de sus clientes... }\end{array}$} \\
\hline Mejoraría & $132(6 \mid .1)$ & $48(72.7)$ & $27(52.9)$ & $27(56.2)$ & $30(58.8)$ & 0.117 \\
\hline Empeoraría / no afectaría & $84(38.9)$ & $18(27.3)$ & $24(47.1)$ & $21(43.8)$ & $2 I(4 \mid .2)$ & \\
\hline \multicolumn{7}{|l|}{$\begin{array}{l}\text { Si su establecimiento fuera libre de humo de tabaco la } \\
\text { salud de sus trabajadores... }\end{array}$} \\
\hline Mejoraría & $142(65.7)$ & $47(7 \mid .2)$ & $35(68.6)$ & $30(62.5)$ & $30(58.8)$ & 0.505 \\
\hline Empeoraría / no afectaría & $74(34.3)$ & $19(28.8)$ & $16(31.4)$ & 18 (37.5) & $2 \mid(4 \mid .2)$ & \\
\hline
\end{tabular}

*Frecuencia (porcentaje)

mitad de los establecimientos apoyaba el que los bares y restaurantes fueran libres de humo, a pesar de reconocer que esto mejoraría la salud de clientes y trabajadores. La mayoría de los establecimientos consideró que la implementación de espacios 100\% libres de humo de tabaco en bares y restaurantes disminuiría el número de clientes.

Comparado con el resto de las ciudades, los dueños y administradores de la Ciudad de México reportaron una mayor prevalencia de establecimientos donde los clientes tenían prohibido fumar, siendo también la ciudad donde se observaron las concentraciones más bajas de nicotina. Esto indica un alto grado de apego con la LPSNF-DF, con $85.4 \%$ de los establecimientos prohibiendo el consumo de tabaco en cualquier área de su establecimiento, mientras que en promedio $15.3 \%$ de los establecimientos en Colima, Cuernavaca y Toluca lo hacía. En dichas ciudades, el reporte de implementación de las políticas se correlacionó adecuadamente con las concentraciones de nicotina ambiental, siendo menores en los establecimientos con prohibición, incrementándose en los establecimientos con áreas para fumar y siendo substancialmente mayores en los establecimientos donde fumar estaba permitido sin restricciones. Es interesante que el cumplimiento con la LPSNF se logre a pesar del menor apoyo de los bares y restaurantes de la Ciudad de México hacia los espacios públicos libres de humo de tabaco, comparado con el resto de las ciudades.

Tras la implementación de la LPSNF-DF la CANIRAC estimó una disminución entre el 10 y el 30\% 


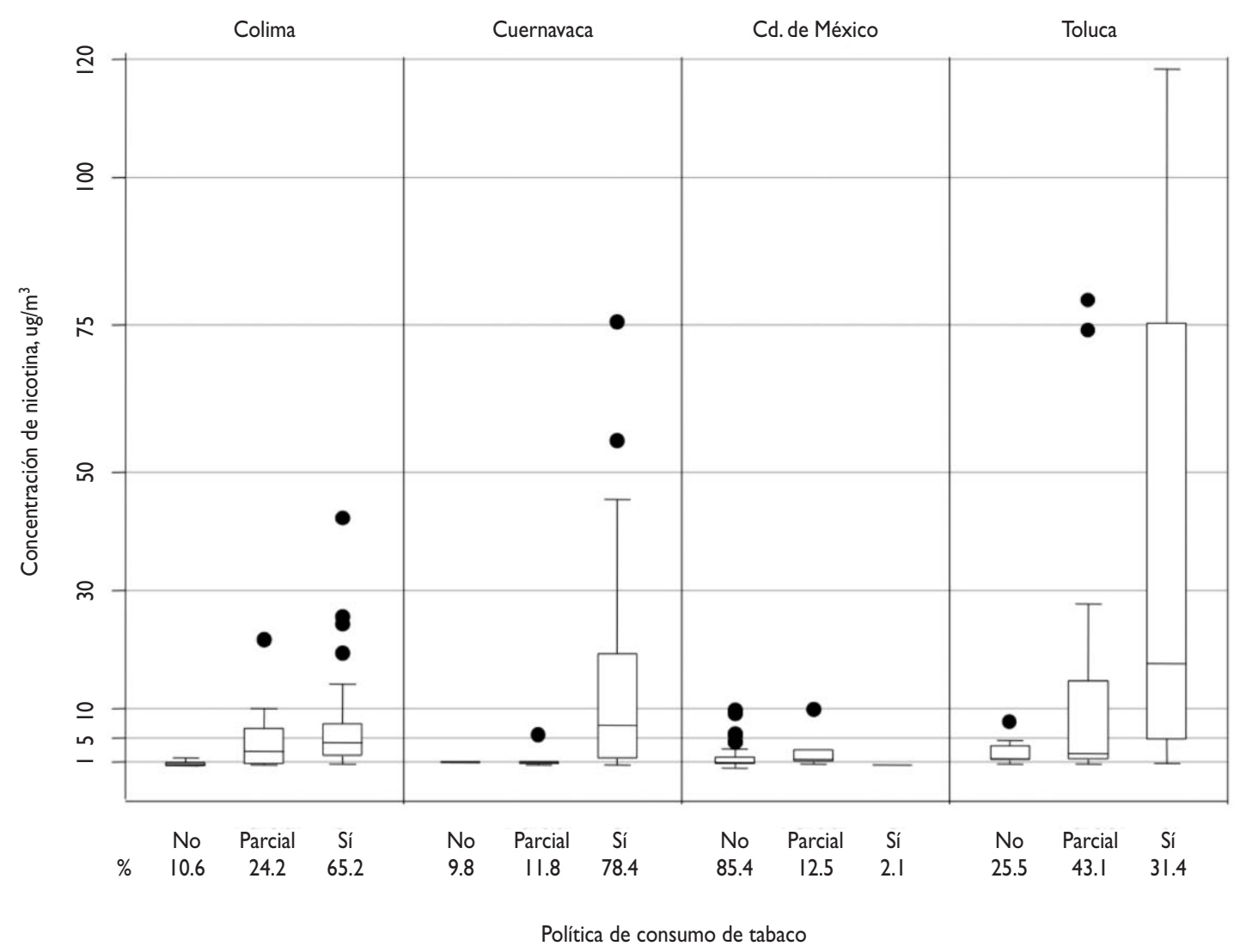

Figura I. CONCENTRACIONES DE NICOTINA AMBIENTAL POR CIUDAD Y POLÍTICA DE CONSUMO DE TABACO IMPLEMENTADA. MÉxıco, 2008

en el ingreso de los establecimientos del sector. ${ }^{19}$ Una disminución de esta magnitud tendría que haber implicado a la mayoría de los establecimientos. En el presente estudio, la disminución en el número de clientes fue reportada por $47.9 \%$ de los administradores de la Ciudad de México, por lo que las pérdidas económicas difícilmente podrían haber sido de la magnitud estimada por la CANIRAC. Este hallazgo concuerda con observaciones previas que han sugerido que la percepción negativa de los bares y restaurantes hacia la implementación de prohibiciones al consumo de tabaco pudiera estar sesgada por las campañas de desinformación de la industria tabacalera y no por pérdidas económicas reales. En México, un estudio reciente que analizó el comportamiento económico del sector hostelero de la Ciudad de México no observó pérdidas tras la implementación de la LPSNF-DF, lo que fortalece la posibilidad de que la preocupación por efectos económicos sea infundada.*

Los resultados de este estudio son consistentes con otras investigaciones, donde se ha detectado apoyo mayoritario y creciente hacia el desarrollo de espacios libres de humo de tabaco en la Ciudad de México, tanto entre la población en general, ${ }^{20,21}$ como entre la población fumadora. ${ }^{15}$ Estudios en otros países coinciden en que el apoyo a los espacios libres de humo se incrementa tras su implementación, incluso entre la población que supuestamente rechazaría la prohibición al consumo de tabaco en áreas interiores, como es la población

\footnotetext{
* Guerrero-López C, Jiménez-Ruiz J, Reynales-Shigematsu LM, Waters $\mathrm{H}$. The economic impact of Mexico City's smokefree law. Tob Control. En prensa.
} 
fumadora $^{22-24}$ y los dueños y trabajadores de bares. ${ }^{25,26}$ La implementación de espacios libres de humo reduce la aceptabilidad social del fumar, ${ }^{27}$ lo que, aunado a campañas de difusión sobre sus beneficios, asegura el cumplimiento y cambio social sustentable. ${ }^{28}$

La interpretación de los resultados debe tener en cuenta el diseño del estudio. El diseño transversal impide establecer causalidad entre la opinión de los dueños y administradores de bares y restaurantes en la Ciudad de México y la implementación de la LPSNF-DF. Sin embargo, el diseño aprovecha el experimento natural generado al implementar la LPSNF-DF únicamente en la Ciudad de México, lo que posibilita la contrastación con ciudades sin prohibición. Los administradores reportaron que sus establecimientos eran, en su mayor parte, de nivel socioeconómico alto, lo que podría sugerir un sesgo de selección. La selección aleatoria de los bares y restaurantes se basó en el registro oficial de establecimientos, que ofrecía el mejor marco muestral disponible, aunque el subregistro de establecimientos con menor ingreso económico es posible. A pesar de estas limitaciones, la selección aleatoria de establecimientos asegura una mayor representatividad de la muestra que cualquier selección a conveniencia, estrategia comúnmente utilizada en este tipo de estudios. ${ }^{29}$

En conclusión, aunque los dueños y los administradores reconocen que la exposición al HTA representa un riesgo a la salud y que es necesario crear espacios $100 \%$ libres de humo de tabaco, existe reticencia a implementar prohibiciones al consumo de tabaco en bares y restaurantes. La preocupación sobre posibles efectos económicos negativos de la prohibición es patente, pudiendo explicar al menos parcialmente el rechazo a la implementación de este tipo de políticas. Considerando que los dueños y administradores de bares y restaurantes son un pilar fundamental para asegurar la eliminación de la exposición al HTA es necesario establecer estrategias de comunicación que permitan el intercambio de información, el diálogo y la toma conjunta de decisiones basadas en evidencia científica sólida.

\section{Agradecimientos}

El presente estudio ha sido financiado a través del Consejo Nacional de Ciencia y Tecnología de México, con el proyecto "Exposición al Humo de Tabaco Ambiental, Salud y Productividad en Bares y Restaurantes de México" (S0008-2007-1-69760); del National Institute of Environmental Health Sciences, a través del Center for Research in Environmental Diseases del MD Anderson Cancer Center (ES007784); y del Pilot Projects Program del National Institute of Occupational Safety and Health, a través del Southwest Center for Occupational and Environmental Health de la Universidad de Texas School of Public Health (T42 OH008421).

\section{Declaración de conflicto de intereses}

Declaramos no tener conflicto de intereses.

\section{Referencias}

I.World Health Organization.WHO Framework Convention on Tobacco Control (WHO FCTC). Geneva, 2003.

2.World Health Organization. Policy recommendations on protection from exposure to second-hand tobacco smoke. Geneva, 2007.

3.Alpert HR, Carpenter CM, Travers MJ, Connolly GN. Environmental and economic evaluation of the Massachusetts Smoke-Free Workplace Law.J Community Health 2007;32(4):269-28I.

4. Goodman P, Agnew M, McCaffrey M, Paul G, Clancy L. Effects of the Irish smoking ban on respiratory health of bar workers and air quality in Dublin pubs. Am J Respir Crit Care Med 2007; 175:840-845.

5. Juster HR, Loomis BR, Hinman TM, Farrelly MC, Hyland A, Bauer UE, et al. Declines in hospital admissions for acute myocardial infarction in New York state after implementation of a comprehensive smoking ban. Am J Public Health 2007;97( I I):2035-2039.

6. Fernandez E, Fu M, Pascual JA, Lopez MJ, Perez-Rios M, Schiaffino A, et al. Impact of the Spanish smoking law on exposure to second-hand smoke and respiratory health in hospitality workers: a cohort study. PLoS One 2009;4(I):e4244.

7.Valente P, Forastiere F, Bacosi A, Cattani G, Di Carlo S, Ferri M, et al. Exposure to fine and ultrafine particles from secondhand smoke in public places before and after the smoking ban, Italy 2005. Br Med J 2007;16(5):312-317.

8. Navas-Acien A, Peruga A, Breysse P, Zavaleta A, Blanco-Marquizo A, Pitarque $R$, et al. Secondhand tobacco smoke in public places in Latin America, 2002-2003. JAMA 2004;29I(22):274I-5.

9. Nebot M, Lopez MJ, Gorini G, Neuberger M,Axelsson S, Pilali M, et al. Environmental tobacco smoke exposure in public places of European cities. Tob Control 2005; |4(I):60-63.

10. Lazcano-Ponce E, Benowitz N, Sanchez-Zamorano LM, BarbosaSanchez L,Valdes-Salgado R, Jacob P, 3rd, et al. Secondhand smoke exposure in Mexican discotheques. Nicotine Tob Res 2007;9(I0): I02I-1026.

II. Siegel M, Skeer M. Exposure to secondhand smoke and excess lung cancer mortality risk among workers in the "5 B's": bars, bowling alleys, billiard halls, betting establishments, and bingo parlours. Tob Control 2003; 12(3):333-338.

12. US Department of Health and Human Services. The Health Consequences of Involuntary Exposure to Tobacco Smoke:A Report of the Surgeon General. Atlanta, GA: US Department of Health and Human Services, 2006

13. Drope J, Bialous SA, Glantz SA. Tobacco industry efforts to present ventilation as an alternative to smoke-free environments in North America. Tob Control 2004; I3(suppl I):i4 I-i47.

14. Gaceta Oficial del Distrito Federal. Decreto por el que se reforman, adicionan y derogan la Ley de Proteccion a la Salud de los No Fumadores del Distrito Federal y la Ley para el Funcionamiento de Establecimientos Mercantiles del Distrito Federal 2008. 4 de marzo de 2008;287.

15. Thrasher J, Besley J, Gonzalez W. Perceived justice and popular support for public health laws: Citizens' perceptions of comprehensive smoke-free legislation in Mexico City. Soc Sci Med 2010;70:787-793. 
16. Scollo M, Lal A, Hyland A, Glantz S. Review of the quality of studies on the economic effects of smoke-free policies on the hospitality industry. Tob Control 2003; 12(1):13-20.

17. Rosas V.Acatan comercios con resignación la Ley antitabaco. El Universal. 3 de abril de 2008.

18. Reynales-Shigematsu LM, Rodriguez-Bolanos R,Valdés-Salgado R, Lazcano-Ponce EC, Hernández-Ávila M. Encuesta de Tabaquismo en Jovenes:Analisis descriptivo 2003, 2005, 2006, 2008. Cuernavaca, México: Instituto Nacional de Salud Publica de México, 2009.

19. Morales-Hernández C. CANIRAC: Los restauranteros del DF están muy golpeados. IQ Magazine. agosto de 2009:47-49.

20.Thrasher J, Sawayampakala K, Arillo-Santillán E, Sebrié E,Walseman K, Bottai M. Differential impact of local and federal smoke-free legislation in Mexico:A longitudinal study of campaign exposure, support for smokefree policies and secondhand tobacco smoke exposure among adult smokers. Salud Publica Mex 2010;52(suppl 2):S242-S25I.

21.Thrasher J, Perez-Hernández R, Sawayamkapala K, Arillo-Santillán E, Bottai M. Translating the World Health Organization's Framework Convention on Tobacco Control: Policy support, norms, and secondhand smoke exposure before and after implementation of a comprehensive smoke-free policy in Mexico City.Am J Public Health. Epub 2010 May 13. 22. Fong G, Hyland A, Borland R, Hammond D, Hastings G, McNeill A, et al. Reductions in tobacco smoke pollution and increases in support for smoke-free public places following the implementation of comprehensive smoke-free workplace legislation in the Republic of Ireland: Findings from the ITC Ireland/UK survey. Br Med J 2006; I5(suppl 3):iii5 I-iii58.

23. Hyland A, Higbee C, Borland R, Travers M, Hastings G, Fong GT, et al. Attitudes and beliefs about secondhand smoke and smoke-free policies in four countries: Findings from the International Tobacco Control Four Country Survey. Nicotine Tobacco Res 2009; I (6):642-649.

24. Thrasher JF, Boado M, Sebrie EM, Bianco E. Smoke-free policies and the social acceptability of smoking in Uruguay and Mexico: Findings from the International Tobacco Control Policy Evaluation Project. Nicotine Tobacco Res 2009; I (6):59I.

25. Hilton S, Semple S, Miller BG, MacCalman L, Petticrew M, Dempsey S, et al. Expectations and changing attitudes of bar workers before and after the implementation of smoke-free legislation in Scotland. BMC Public Health 2007;7(206): I 47I-2458.

26. Tang H, Cowling D, Stevens C, Lloyd J. Changes of knowledge, attitudes, beliefs, and preference of bar owner and staff in response to a smoke-free bar law. Br Med J 2004; I3(I):87-89.

27.Thrasher JF, Reynales-Shigematsu LM, Baezconde-Garbanati L, Villalobos V,Téllez-Girón P,Arillo-Santillán E, et al. Promoting the Effective Translation of the Framework Convention on Tobacco Control. Eval Health Prof 2008;3I(2): I45-I66

28.Villalobos V, Ortiz-Ramírez O,Thrasher J, Pérez-Hernández R, Cedillo C, Arillo-Santillán E. Mercadotecnia social para promover la salud pública: el desarrollo de una campaña para promover espacios $100 \%$ libres de humo de tabaco. Salud Publica Mex 20I0;52(suppl 2):SI27-SI 35.

29. Barrientos-Gutiérrez T,Valdés-Salgado R, Reynales-Shigematsu LM, Navas-Acien A, Lazcano-Ponce EC. Exposición involuntaria al humo de tabaco en lugares públicos de la Ciudad de México. Salud Publica Mex 2007;43(supl. 2):S205-S2I2. 